Pacific

Journal of

Mathematics

\title{
ELLIPTIC FUNCTIONS TO THE QUINTIC BASE
}

Heng Huat Chan and Zhi-Guo LiU 


\title{
ELLIPTIC FUNCTIONS TO THE QUINTIC BASE
}

\author{
Heng Huat Chan AND Zhi-Guo LiU
}

\begin{abstract}
We study two elliptic functions to the quintic base and find two nonlinear second order differential equations satisfied by them. We then derive two recurrence relations involving certain Eisenstein series associated with the group $\Gamma_{0}(5)$. These recurrence relations allow us to derive infinite families of identities involving the Eisenstein series and Dedekind $\eta$-products. An imaginary transformation for one of the elliptic functions is also derived.
\end{abstract}

\section{Introduction}

Let $q=e^{2 \pi i \tau}$, where $\operatorname{Im} \tau>0$. The Dedekind $\eta$-function is defined by

$$
\eta(\tau)=q^{1 / 24}(q ; q)_{\infty}
$$

where

$$
(a ; q)_{\infty}=\prod_{n=1}^{\infty}\left(1-a q^{n-1}\right) .
$$

Srinivasa Ramanujan, on a page of his $\tau(n)-p(n)$ manuscript [Ramanujan 1988, p. 139; Berndt and Ono 1999], stated without proof that

$$
\sum_{n=1}^{\infty}\left(\frac{n}{5}\right) \frac{q^{n}}{\left(1-q^{n}\right)^{2}}=\frac{\eta^{5}(5 \tau)}{\eta(\tau)},
$$

where $(-)$ is the Legendre symbol.

This identity is of great interest historically because Ramanujan deduced from it his famous partition identity

$$
\sum_{n=0}^{\infty} p(5 n+4) q^{n}=5 \frac{\left(q^{5} ; q^{5}\right)_{\infty}^{5}}{(q ; q)_{\infty}^{6}},
$$

where $p(n)$ is the number of partitions of $n$. Proofs of (1-1) can be found in [Bailey 1952a; 1952b], and more recently in [Shen 1994, p. 329; Chan 1995].

MSC2000: 33E05, 11F11, 11F27.

Keywords: Eisenstein series, elliptic functions, Dedekind eta function.

Liu was supported in part by Shanghai Priority Academic Discipline and the National Science Foundation of China. 
In an unpublished work, Z.-G. Liu and R. P. Lewis discovered that

$$
\sum_{n=1}^{\infty} \frac{n^{3}\left(q^{n}-q^{2 n}-q^{3 n}+q^{4 n}\right)}{\left(1-q^{5 n}\right)}=\frac{\eta^{5}(5 \tau)}{\eta(\tau)} A(\tau),
$$

where

$$
\begin{aligned}
A(\tau) & =1+6 \sum_{n \geq 1} \frac{n q^{n}}{1-q^{n}}-30 \sum_{n \geq 1} \frac{n q^{5 n}}{1-q^{5 n}} \\
& =\frac{\eta^{5}(\tau)}{\eta(5 \tau)} \sqrt{1+22\left(\frac{\eta(5 \tau)}{\eta(\tau)}\right)^{6}+125\left(\frac{\eta(5 \tau)}{\eta(\tau)}\right)^{12}} .
\end{aligned}
$$

The last identity relating $A(\tau)$ to $\eta$-products can be found in [Berndt et al. 2000, Lemma 3.6].

If we rewrite the left-hand side of (1-1) as

$$
\sum_{n=1}^{\infty}\left(\frac{n}{5}\right) \frac{q^{n}}{\left(1-q^{n}\right)^{2}}=\sum_{n=1}^{\infty} \frac{n\left(q^{n}-q^{2 n}-q^{3 n}+q^{4 n}\right)}{\left(1-q^{5 n}\right)}
$$

and set

$$
L_{k}(q)=\sum_{n=1}^{\infty} \frac{n^{k}\left(q^{n}-q^{2 n}-q^{3 n}+q^{4 n}\right)}{\left(1-q^{5 n}\right)},
$$

then we observe that (1-1) and (1-2) correspond to expressions in terms of the Dedekind $\eta$-products for $L_{k}(q)$ when $k=1$ and 3 respectively.

Motivated by our recent paper [Chan and Liu 2003], we propose to construct a recurrence satisfied by $L_{2 k+1}(q)$, which would then give us expressions of $L_{2 k+1}(q)$ in terms of $\eta$ products for every integer $k \geq 0$. In order to achieve this, we first define

$$
V(u \mid \tau)=4 \sum_{k=0}^{\infty}(-1)^{k} \frac{(2 u)^{2 k+1}}{(2 k+1) !} L_{2 k+1}(q) .
$$

In Section 2, we will see that $V(u \mid \tau)$ is a elliptic function with periods $\pi$ and $5 \pi \tau$. In Sections 3 and 4, we construct two differential equations associated with $V(u \mid \tau)$ and

$$
M(u \mid \tau):=\frac{4}{3}\left(A(\tau)+6 \sum_{k=1}^{\infty} \frac{(-1)^{k}(2 u)^{2 k}}{(2 k) !} S_{2 k+1}(q)\right),
$$

where $A(\tau)$ is given by $(1-3)$ and

$$
S_{k}(q)=\sum_{n \geq 1} \frac{n^{k}\left(q^{n}+q^{2 n}+q^{3 n}+q^{4 n}\right)}{1-q^{5 n}} .
$$


Using these differential equations, we obtain two difference equations satisfied by $S_{2 k+1}(q)$ and $L_{2 k+1}(q)$. These equations allow us to tabulate $S_{2 k+1}(q)$ and $L_{2 k+1}(q)$ in terms of the modular functions

$$
x=\left(\frac{\eta(5 \tau)}{\eta(\tau)}\right)^{6} \quad \text { and } \quad z=\frac{\eta^{5}(\tau)}{\eta(5 \tau)}
$$

for any $k \geq 1$. In Section 5, we study the behavior of $V(u \mid \tau)$ under the imaginary transformation $\tau \mapsto-1 /(5 \tau)$. As a corollary, we indicate some computations of special values of Dirichlet $L$-series.

\section{The Jacobi theta function $\vartheta_{1}(u \mid \tau)$ and $V(u \mid \tau)$}

Recall that the Jacobi theta functions $\vartheta_{1}(u \mid \tau)$ and $\vartheta_{4}(u \mid \tau)$ are given by

$$
\begin{aligned}
& \vartheta_{1}(u \mid \tau)=2 q^{1 / 8} \sum_{n \geq 0}(-1)^{n} q^{n(n+1) / 2} \sin (2 n+1) u, \\
& \vartheta_{4}(u \mid \tau)=1+2 \sum_{n \geq 1}^{\infty}(-1)^{n} q^{n^{2} / 2} \cos 2 n u .
\end{aligned}
$$

From [Whittaker and Watson 1927, p. 489] we know that

$$
\frac{\vartheta_{4}^{\prime}}{\vartheta_{4}}(u \mid \tau)=4 \sum_{n \geq 1} \frac{q^{n / 2}}{1-q^{n}} \sin 2 n u .
$$

Hence,

$$
\begin{aligned}
\frac{\vartheta_{4}^{\prime}}{\vartheta_{4}}\left(u+\frac{3}{2} \pi \tau \mid 5 \tau\right) & +\frac{\vartheta_{4}^{\prime}}{\vartheta_{4}}\left(u-\frac{3}{2} \pi \tau \mid 5 \tau\right) \\
& =4 \sum_{n \geq 1} \frac{q^{5 n / 2}}{1-q^{5 n}}\left(\sin 2 n\left(u+\frac{3}{2} \pi \tau\right)+\sin 2 n\left(u-\frac{3}{2} \pi \tau\right)\right) \\
& =8 \sum_{n \geq 1} \frac{q^{5 n / 2}}{1-q^{5 n}} \cos 3 n \pi \tau \sin 2 n u \\
& =4 \sum_{n \geq 1} \frac{q^{5 n / 2}\left(q^{3 n / 2}+q^{-3 n / 2}\right)}{1-q^{5 n}} \sin 2 n u=4 \sum_{n \geq 1} \frac{q^{n}+q^{4 n}}{1-q^{5 n}} \sin 2 n u .
\end{aligned}
$$

Similarly,

$$
\frac{\vartheta_{4}^{\prime}}{\vartheta_{4}}\left(u+\frac{1}{2} \pi \tau \mid 5 \tau\right)+\frac{\vartheta_{4}^{\prime}}{\vartheta_{4}}\left(u-\frac{1}{2} \pi \tau \mid 5 \tau\right)=4 \sum_{n \geq 1} \frac{q^{2 n}+q^{3 n}}{1-q^{5 n}} \sin 2 n u .
$$


This implies that

(2-1) $\quad V(u \mid \tau)=\frac{\vartheta_{4}^{\prime}}{\vartheta_{4}}\left(u+\frac{3}{2} \pi \tau \mid 5 \tau\right)+\frac{\vartheta_{4}^{\prime}}{\vartheta_{4}}\left(u-\frac{3}{2} \pi \tau \mid 5 \tau\right)$

$$
-\frac{\vartheta_{4}^{\prime}}{\vartheta_{4}}\left(u+\frac{1}{2} \pi \tau \mid 5 \tau\right)-\frac{\vartheta_{4}^{\prime}}{\vartheta_{4}}\left(u-\frac{1}{2} \pi \tau \mid 5 \tau\right),
$$

where $V(u \mid \tau)$ is given by (1-4). By using the transformation formulas

$$
\frac{\vartheta_{1}^{\prime}}{\vartheta_{1}}(u \mid \tau)=-i+\frac{\vartheta_{4}^{\prime}}{\vartheta_{4}}\left(u-\frac{1}{2} \pi \tau \mid \tau\right), \quad \frac{\vartheta_{1}^{\prime}}{\vartheta_{1}}(u-\pi \tau \mid \tau)=\frac{\vartheta_{1}^{\prime}}{\vartheta_{1}}(u \mid \tau)-2 i,
$$

we find that

$$
\begin{aligned}
\frac{\vartheta_{1}^{\prime}}{\vartheta_{1}}(u+\pi \tau \mid 5 \tau)+\frac{\vartheta_{1}^{\prime}}{\vartheta_{1}}(u-\pi \tau \mid 5 \tau) & =\frac{\vartheta_{4}^{\prime}}{\vartheta_{4}}\left(u+\frac{3}{2} \pi \tau \mid 5 \tau\right)+\frac{\vartheta_{4}^{\prime}}{\vartheta_{4}}\left(u-\frac{3}{2} \pi \tau \mid 5 \tau\right) \\
& =4 \sum_{n \geq 1} \frac{q^{n}+q^{4 n}}{1-q^{5 n}} \sin 2 n u, \\
\frac{\vartheta_{1}^{\prime}}{\vartheta_{1}}(u+2 \pi \tau \mid 5 \tau)+\frac{\vartheta_{1}^{\prime}}{\vartheta_{1}}(u-2 \pi \tau \mid 5 \tau) & =\frac{\vartheta_{4}^{\prime}}{\vartheta_{4}}\left(u+\frac{1}{2} \pi \tau \mid 5 \tau\right)+\frac{\vartheta_{4}^{\prime}}{\vartheta_{4}}\left(u-\frac{1}{2} \pi \tau \mid 5 \tau\right) \\
& =4 \sum_{n \geq 1} \frac{q^{2 n}+q^{3 n}}{1-q^{5 n}} \sin 2 n u .
\end{aligned}
$$

Combining formulas (2-1) through (2-3), we conclude that

$$
\begin{aligned}
V(u \mid \tau)=\frac{\vartheta_{1}^{\prime}}{\vartheta_{1}}(u+\pi \tau \mid 5 \tau)+\frac{\vartheta_{1}^{\prime}}{\vartheta_{1}}(u-\pi \tau \mid 5 \tau) & \\
& -\frac{\vartheta_{1}^{\prime}}{\vartheta_{1}}(u+2 \pi \tau \mid 5 \tau)-\frac{\vartheta_{1}^{\prime}}{\vartheta_{1}}(u-2 \pi \tau \mid 5 \tau) .
\end{aligned}
$$

\section{Differential equations satisfied by $V(u \mid \tau)$ and $M(u \mid \tau)$}

Before we proceed with the derivation of a differential equation satisfied by $V(u \mid \tau)$, we first define the classical Eisenstein series to be used in the sequel. For $\operatorname{Im} \tau>0$, the Eisenstein series $E_{2}(\tau)$ is given by

$$
E_{2}(\tau)=1-24 \sum_{n \geq 1} \frac{n q^{n}}{1-q^{n}}, q=e^{2 \pi i \tau} .
$$

For integer $k \geq 2$, we define

$$
E_{2 k}(\tau)=1+\frac{(2 \pi i)^{2 k}}{(2 k-1) ! \zeta(2 k)} \sum_{n \geq 1} \frac{n^{2 k-1} q^{n}}{1-q^{n}},
$$

where

$$
\zeta(s)=\sum_{n \geq 1} \frac{1}{n^{s}}, \quad \operatorname{Re} s>1 .
$$


We now construct a differential equation satisfied by $V(u \mid \tau)$. Define

$$
f(v):=f(v \mid \tau)=\frac{\begin{array}{l}
\vartheta_{1}(v+u+\pi \tau \mid 5 \tau) \vartheta_{1}(v+u-\pi \tau \mid 5 \tau) \\
\times \vartheta_{1}(v-u+2 \pi \tau \mid 5 \tau) \vartheta_{1}(v-u-2 \pi \tau \mid 5 \tau)
\end{array}}{\vartheta_{1}^{4}(v \mid 5 \tau)},
$$

where $|u|<\pi|\tau|$. Our choice of $f(v \mid \tau)$ is clearly motivated by (2-4). Using the transformation formulas

$$
\vartheta_{1}(t+\pi \mid \tau)=-\vartheta_{1}(t \mid \tau) \text { and } \vartheta_{1}(t+\pi \tau \mid \tau)=-q^{-1 / 2} e^{-2 i t} \vartheta_{1}(t \mid \tau)
$$

from [Whittaker and Watson 1927, p. 463], we verify that $f(v)$ is an elliptic function with periods $\pi$ and $5 \pi \tau$. It has a pole of order 4 at $v=0$. Now, set $F(v)=v^{4} f(v)$ and

$$
\phi(v)=\frac{F^{\prime}(v)}{F(v)} .
$$

By elementary calculations, we find that

$$
\operatorname{res}(f(v) ; v=0)=\frac{1}{6} F(0)\left(\phi^{3}(0)+3 \phi(0) \phi^{\prime}(0)+\phi^{\prime \prime}(0)\right),
$$

where $\operatorname{res}(f(v) ; v=p)$ denotes the residue of $f$ at the pole $p$. Since the sum of residues of an elliptic function over a period parallelogram is zero and $F(0) \neq 0$, we conclude that

$$
\phi^{3}(0)+3 \phi(0) \phi^{\prime}(0)+\phi^{\prime \prime}(0)=0 .
$$

Next, note that

$$
\begin{aligned}
\phi(v)=\frac{4}{v}-4 \frac{\vartheta_{1}^{\prime}}{\vartheta_{1}}(u \mid 5 \tau)+\frac{\vartheta_{1}^{\prime}}{\vartheta_{1}}(v+u+\pi \tau \mid 5 \tau)+\frac{\vartheta_{1}^{\prime}}{\vartheta_{1}}(v+u-\pi \tau \mid 5 \tau) \\
+\frac{\vartheta_{1}^{\prime}}{\vartheta_{1}}(v-u+2 \pi \tau \mid 5 \tau)+\frac{\vartheta_{1}^{\prime}}{\vartheta_{1}}(v-u-2 \pi \tau \mid 5 \tau) \\
=\frac{4}{3} E_{2}(5 \tau) v+\frac{\vartheta_{1}^{\prime}}{\vartheta_{1}}(v+u+\pi \tau \mid 5 \tau)+\frac{\vartheta_{1}^{\prime}}{\vartheta_{1}}(v+u-\pi \tau \mid 5 \tau) \\
+\frac{\vartheta_{1}^{\prime}}{\vartheta_{1}}(v-u+2 \pi \tau \mid 5 \tau)+\frac{\vartheta_{1}^{\prime}}{\vartheta_{1}}(v-u-2 \pi \tau \mid 5 \tau)+O\left(v^{3}\right),
\end{aligned}
$$

where we have used the expansion

$$
\frac{\vartheta_{1}^{\prime}}{\vartheta_{1}}(t \mid \tau)=\cot t+4 \sum_{n \geq 1} \frac{q^{n}}{1-q^{n}} \sin 2 n t
$$

from [Whittaker and Watson 1927, p. 489] and the representation given in (3-1). From (3-4), we find that

$$
\phi(0)=V(u \mid \tau) .
$$


Differentiating (3-4) with respect to $v$ and setting $v=0$, we find that (3-6) $\begin{aligned} \phi^{\prime}(0)=\frac{4}{3} E_{2}(5 \tau)+\left(\frac{\vartheta_{1}^{\prime}}{\vartheta_{1}}\right)^{\prime}(u+\pi \tau \mid 5 \tau)+\left(\frac{\vartheta_{1}^{\prime}}{\vartheta_{1}}\right)^{\prime}(u-\pi \tau \mid 5 \tau) \\ +\left(\frac{\vartheta_{1}^{\prime}}{\vartheta_{1}}\right)^{\prime}(u+2 \pi \tau \mid 5 \tau)+\left(\frac{\vartheta_{1}^{\prime}}{\vartheta_{1}}\right)^{\prime}(u-2 \pi \tau \mid 5 \tau) .\end{aligned}$

Differentiating both sides of (2-2) and (2-3), we conclude from (3-6) that

$$
M(u \mid \tau)=\phi^{\prime}(0),
$$

where $M(u \mid \tau)$ is given by (1-5). Finally differentiating (3-4) twice and setting $v=0$, we conclude that

$$
\phi^{\prime \prime}(0)=V^{\prime \prime}(u \mid \tau)
$$

by (2-4). Substituting these expressions for $\phi(0), \phi^{\prime}(0)$ and $\phi^{\prime \prime}(0)$ into (3-3), we conclude:

Theorem 3.1.

$$
V^{\prime \prime}(u \mid \tau)+3 M(u \mid \tau) V(u \mid \tau)+V^{3}(u \mid \tau)=0 .
$$

Corollary 3.2. For integer $k \geq 1$,

$$
\begin{aligned}
L_{2 k+1}=A L_{2 k-1}+6 \sum_{l=1}^{k-1} & \frac{(2 k-1) ! L_{2(k-l-1)+1} S_{2 l+1}}{(2 l) !(2(k-l)-1) !} \\
& \quad-4 \sum_{l=0}^{k-1} \sum_{m=0}^{k-l-2} \frac{(2 k-1) ! L_{2 l+1} L_{2 m+1} L_{2(k-l-m-2)+1}}{(2 l+1) !(2 m+1) !(2(k-l-m-2)+1) !},
\end{aligned}
$$

where $L_{k}:=L_{k}(q)$ and $S_{k}:=S_{k}(q)$.

The recurrence in the corollary involves both $L_{k}$ and $S_{k}$. In order to tabulate $L_{k}$, we need another difference equation expressing $S_{k}$ in terms of $L_{s}$ and $S_{t}$ for $3 \leq s, t<k$. To achieve this, we need to obtain a second differential equation satisfied by $M:=M(u \mid \tau)$ and $V:=V(u \mid \tau)$. Using the method in the proof of Theorem 3.1 with $f$ replaced by

$$
\begin{aligned}
g(v)=\frac{\vartheta_{1}(2 v \mid 5 \tau)}{\vartheta_{1}^{8}(v \mid 5 \tau)} \vartheta_{1}(v+u+\pi \tau \mid 5 \tau) & \vartheta_{1}(v+u-\pi \tau \mid 5 \tau) \\
& \times \vartheta_{1}(v-u+2 \pi \tau \mid 5 \tau) \vartheta_{1}(v-u-2 \pi \tau \mid 5 \tau),
\end{aligned}
$$

we find that

$$
\begin{aligned}
V^{6}+ & 14 V^{4} M+60 M V^{(2)} V+15 M^{3}-\frac{128}{9} E_{6}(5 \tau)+6 V^{(4)} V \\
+ & 20 V^{(2)} V^{3}+10\left(V^{\prime \prime}\right)^{2}+45 M^{2} V^{2}+15\left(-\frac{16}{15} E_{4}(5 \tau)+M^{(2)}\right)\left(M+V^{2}\right)=0,
\end{aligned}
$$

where $E_{4}(\tau)$ and $E_{6}(\tau)$ are given by (3-2) and ${ }^{(i)}$ denotes $i$-fold partial differentiation with respect to $u$. 
It turns out that there is a differential equation simpler than the one just given:

$$
M^{(2)}-\frac{8}{3} E_{6}(5 \tau)+\frac{3}{2} M^{2}+3\left(M-\frac{4}{3} A\right) V^{2}+3 V^{(2)} V+\frac{3}{2}\left(V^{\prime}\right)^{2}=0 .
$$

We will devote the next section to the proof this equation. In the meantime, here is a result that follows immediately from (3-7):

Corollary 3.3. For $k \geq 2$,

$$
\begin{aligned}
S_{2 k+1}=A S_{2 k-1}+ & 6 L_{1} L_{2 k-1}+6 \sum_{l=1}^{k-1} \frac{(2 k-2) ! L_{2 l+1} L_{2(k-l-1)+1}}{(2 l-1) !(2(k-l-1)+1) !} \\
+ & 3 \sum_{l=1}^{k-2} \frac{(2 k-2) !\left(S_{2(k-l-1)+1} S_{2 l+1}+L_{2 l+1} L_{2(k-l-1)+1}\right)}{(2 l) !(2(k-l-1)) !} \\
& -12 \sum_{l=0}^{k-3} \sum_{m=0}^{k-l-3} \frac{(2 k-2) ! L_{2 l+1} L_{2 m+1} S_{2(k-l-m-2)+1}}{(2 l+1) !(2 m+1) !(2(k-l-m-2)) !} .
\end{aligned}
$$

Alternate applications of Corollaries 3.2 and 3.3 yield tables for $L_{2 k+1}$ and $S_{2 k+1}$ in terms of $z$ and $x$ given by (1-6). Here are first few terms derived from these recurrences:

$$
\begin{array}{ll}
L_{1}(q)=z x, & S_{3}(q)=z^{2}\left(x+13 x^{2}\right), \\
L_{3}(q)=z x A(\tau), & S_{5}(q)=z^{2} A(\tau)\left(x+31 x^{2}\right), \\
L_{5}(q)=z^{3} x\left(1+40 x+335 x^{2}\right), & S_{7}(q)=z^{4} x\left(1+143 x+3255 x^{2}+20345 x^{3}\right) .
\end{array}
$$

\section{Sketch of the proof of $(3-7)$}

We first note that for $k \geq 1$,

$$
\begin{aligned}
S_{2 k+1}(q) & =\sum_{n \geq 1} \frac{n^{2 k+1} q^{5 n}}{1-q^{5 n}}-\sum_{n \geq 1} \frac{n^{2 k+1} q^{n}}{1-q^{n}} \\
& =\frac{(-1)^{k+1}(2 k+1) ! \zeta(2 k+2)\left(E_{2 k+2}(5 \tau)-E_{2 k+2}(\tau)\right)}{(2 \pi)^{2 k+2}},
\end{aligned}
$$

where $E_{2 k}(\tau)$ is given by (3-2). Ramanujan [Berndt et al. 2000, Theorems 3.1, 3.2] offered the identities expressing $E_{4}(\tau), E_{4}(5 \tau), E_{6}(\tau)$ and $E_{6}(5 \tau)$ in terms of $x, z$ and $A$, and since $E_{2 k}(\tau)$ can be expressed in terms of $E_{4}(\tau)$ and $E_{6}(\tau)$ by [Serre 1973, Corollary 2, p. 89], we can compute $S_{2 k+1}(q)$ in terms of $x, z$, and $A(\tau)$ for any positive integer $k$, and in particular for $1 \leq k \leq 9$. Now, using Corollary 3.2, we compute $L_{2 k+1}(q)$ in terms of $x, z$ and $A$ for $0 \leq k \leq 8$ using the identity

$$
A^{2}(\tau)=z^{2}\left(1+22 x+125 x^{2}\right)
$$


which follows from (1-3) and the definitions of $x$ and $z$ in (1-6). These yield a total of 18 identities, which gives $L_{2 s+1}$ and $S_{2 t+1}$ in terms of $x, z$ and $A(\tau)$ for $0 \leq s \leq 8$ and $1 \leq t \leq 9$.

The coefficients of $u^{2 k+1}$ in $V(u \mid \tau)$ and $u^{2 k}$ in $M(u \mid \tau)$ are constant multiples of $L_{2 k+1}$ and $S_{2 k+1}$ respectively, by (1-4) and (1-5). Hence, we may write

$$
\begin{aligned}
& V(u \mid \tau)=\sum_{k=0}^{8} F_{k}(x, z, A(\tau)) u^{2 k+1}+\mathrm{O}\left(u^{19}\right), \\
& M(u \mid \tau)=\frac{4}{3} A(\tau)+\sum_{k=1}^{9} G_{k}(x, z, A(\tau)) u^{2 k}+\mathrm{O}\left(u^{20}\right),
\end{aligned}
$$

where $F_{k}$ and $G_{k}$ are certain polynomials in $x, z$ and $A(\tau)$.

Identity (3-7) is discovered by first assuming that there is a relation between $M^{(2)}+\alpha_{1} E_{6}(5 \tau), M^{2},\left(M+\alpha_{2} A\right) V^{2}, V^{(2)} V$, and $\left(V^{\prime}\right)^{2}$, namely,

$$
M^{(2)}+\alpha_{1} E_{6}(5 \tau)+\beta_{1} M^{2}+\beta_{2}\left(M+\alpha_{2} A\right) V^{2}+\beta_{3} V^{(2)} V+\beta_{4}\left(V^{\prime}\right)^{2}=0 .
$$

We then use the representations (4-2) to establish relations among $\beta_{i}$ 's by equating the coefficients of $u^{k}$. The determination of $\beta_{i}$ 's gives (3-7) immediately.

In order to show that (3-7) is valid, we note that $M$ and $V$ are both elliptic functions with the total number of poles being 8 and 4 respectively; see (2-4) and (3-6). Therefore the left-hand side $h(u)$ of (3-7) is an elliptic function with at most 16 poles. We know that an elliptic function has the same number of poles and zeros in a period parallelogram. Hence, $h(u)$ must have at most 16 zeros in a period parallelogram. This last step can be established by using the representations (4-2) and showing that $h(u)=\mathrm{O}\left(u^{17}\right)$.

\section{Behavior of $V(u \mid \tau)$ under the imaginary transformation $\tau \mapsto-\frac{1}{5 \tau}$}

Let

$U(u \mid \tau)=\frac{\vartheta_{1}^{\prime}}{\vartheta_{1}}\left(u+\frac{\pi}{5} \mid \tau\right)+\frac{\vartheta_{1}^{\prime}}{\vartheta_{1}}\left(u-\frac{\pi}{5} \mid \tau\right)-\frac{\vartheta_{1}^{\prime}}{\vartheta_{1}}\left(u+\frac{2 \pi}{5} \mid \tau\right)-\frac{\vartheta_{1}^{\prime}}{\vartheta_{1}}\left(u-\frac{2 \pi}{5} \mid \tau\right)$.

Theorem 5.1. The function $U(u \mid \tau)$ and $V(u \mid \tau)$ satisfy the transformation formula

$$
V\left(\frac{u}{\tau} \mid-\frac{1}{5 \tau}\right)=\tau U(u \mid \tau)
$$

Proof. The function $\frac{\vartheta_{1}^{\prime}}{\vartheta_{1}}(u \mid \tau)$ satisfies the relation [Chan and Liu 2003, (4.2)]

$$
\frac{\vartheta_{1}^{\prime}}{\vartheta_{1}}\left(\frac{u}{\tau} \mid-\frac{1}{\tau}\right)=\frac{2 i u}{\pi}+\tau \frac{\vartheta_{1}^{\prime}}{\vartheta_{1}}(u \mid \tau)
$$


Replacing $u$ by $u+\frac{\pi}{5}$ and $u+\frac{2 \pi}{5}$ in (5-1), we find that

$$
\begin{aligned}
& \frac{\vartheta_{1}^{\prime}}{\vartheta_{1}}\left(\frac{u}{\tau}+\frac{\pi}{5 \tau} \mid-\frac{1}{\tau}\right)=\frac{2 i u}{\pi}+\frac{2 i}{5}+\tau \frac{\vartheta_{1}^{\prime}}{\vartheta_{1}}\left(u+\frac{\pi}{5} \mid \tau\right) \text { and } \\
& \frac{\vartheta_{1}^{\prime}}{\vartheta_{1}}\left(\frac{u}{\tau}+\frac{2 \pi}{5 \tau} \mid-\frac{1}{\tau}\right)=\frac{2 i u}{\pi}+\frac{4 i}{5}+\tau \frac{\vartheta_{1}^{\prime}}{\vartheta_{1}}\left(u+\frac{2 \pi}{5} \mid \tau\right) .
\end{aligned}
$$

Replacing $u$ by $-u$ in these two equations and using the fact that $\frac{\vartheta_{1}^{\prime}}{\vartheta_{1}}(u \mid \tau)$ is an odd function of $u$ (see (3-5)), we find that

$$
\begin{aligned}
& \frac{\vartheta_{1}^{\prime}}{\vartheta_{1}}\left(\frac{u}{\tau}-\frac{\pi}{5 \tau} \mid-\frac{1}{\tau}\right)=\frac{2 i u}{\pi}-\frac{2 i}{5}+\tau \frac{\vartheta_{1}^{\prime}}{\vartheta_{1}}\left(u-\frac{\pi}{5} \mid \tau\right) \text { and } \\
& \frac{\vartheta_{1}^{\prime}}{\vartheta_{1}}\left(\frac{u}{\tau}-\frac{2 \pi}{5 \tau} \mid-\frac{1}{\tau}\right)=\frac{2 i u}{\pi}-\frac{4 i}{5}+\tau \frac{\vartheta_{1}^{\prime}}{\vartheta_{1}}\left(u-\frac{2 \pi}{5} \mid \tau\right) .
\end{aligned}
$$

From these four equations we deduce Theorem 5.1 immediately.

Let $\chi_{5}(n)=\left(\frac{n}{5}\right)$ and define

$$
E_{2 r, \chi_{5}}(q)=1+(-1)^{r} \frac{\sqrt{5}}{(2 r-1) ! L\left(2 r, \chi_{5}\right)}\left(\frac{2 \pi}{5}\right)^{2 r} \sum_{n \geq 1} \chi_{5}(n) \frac{n^{2 r-1} q^{n}}{1-q^{n}},
$$

where

$$
L\left(r, \chi_{5}\right)=\sum_{n \geq 1} \frac{\chi_{5}(n)}{n^{r}}
$$

Our function $U(u \mid \tau)$ can be expressed in terms of $E_{2 r, \chi_{5}}(q)$ :

Theorem 5.2. $U(u \mid \tau)=-2 \sum_{r \geq 1} u^{2 r-1}\left(\frac{5}{\pi}\right)^{2 r} L\left(2 r, \chi_{5}\right) E_{2 r, \chi_{5}}(q)$.

Proof. Using (3-5), we find that

where

$$
U(u \mid \tau)=S(u)+4 \sum_{n \geq 1} S_{n}(u) \frac{q^{n}}{1-q^{n}},
$$

$$
\begin{aligned}
S(u) & =\cot \left(u+\frac{\pi}{5}\right)+\cot \left(u-\frac{\pi}{5}\right)-\cot \left(u+\frac{2 \pi}{5}\right)-\cot \left(u-\frac{2 \pi}{5}\right), \\
S_{n}(u) & =\sin \left(2 n\left(u+\frac{\pi}{5}\right)\right)+\sin \left(2 n\left(u-\frac{\pi}{5}\right)\right) \\
& \quad-\sin \left(2 n\left(u+\frac{2 \pi}{5}\right)\right)-\sin \left(2 n\left(u-\frac{2 \pi}{5}\right)\right) \\
= & \sqrt{5} \chi_{5}(n) \sin 2 n u .
\end{aligned}
$$

Here the last equality follows by elementary calculations using trigonometric identities. 
From the expansion $\cot u=\sum_{m \geq 1}\left(\frac{1}{u-m \pi}+\frac{1}{u+(m-1) \pi}\right)$, we then find that (5-3) $S(u)=\sum_{k=1}^{4} \chi_{5}(k) \cot \left(u+\frac{k \pi}{5}\right)$

$$
\begin{aligned}
& =\sum_{m \geq 1} \sum_{k=1}^{4} \chi_{5}(k)\left(\frac{1}{u+k \pi / 5-m \pi}+\frac{1}{u+k \pi / 5-(m-1) \pi}\right) \\
& =\sum_{r \geq 0} u^{r}\left(\frac{5}{\pi}\right)^{r+1} \sum_{m \geq 1} \sum_{k=1}^{4} \chi_{5}(k)\left(-\frac{1}{(5 m-k)^{r+1}}+\frac{(-1)^{r}}{(5(m-1)+k)^{r+1}}\right) .
\end{aligned}
$$

Next, we find that

$$
\sum_{m \geq 1} \sum_{k=1}^{4} \frac{\chi_{5}(k)}{(5 m-k)^{r+1}}=\sum_{m \geq 1} \sum_{k=1}^{4} \frac{\chi_{5}(5 m-k)}{(5 m-k)^{r+1}}=\sum_{m \geq 1} \frac{\chi_{5}(n)}{n^{r+1}}=L\left(r+1, \chi_{5}\right)
$$

and

$$
\sum_{m \geq 1} \sum_{k=1}^{4} \frac{\chi_{5}(k)}{(5(m-1)+k)^{r+1}}=L\left(r+1, \chi_{5}\right) .
$$

Hence, we conclude from (5-3) that

$$
S(u)=-2 \sum_{r \geq 0} u^{2 r+1}\left(\frac{5}{\pi}\right)^{2 r+2} L\left(2 r+2, \chi_{5}\right)
$$

and this completes the proof of Theorem 5.2.

Now, using Theorem 5.2 and equating the coefficients of powers of $u$ in Theorem 5.1, we derive a transformation formula for $L_{2 k+1}$ and $E_{2 k+1, \chi_{5}}$ :

\section{Theorem 5.3.}

$$
L\left(2 k+2, \chi_{5}\right) E_{2 k+2, \chi_{5}}\left(e^{2 \pi i \tau}\right)=\frac{(-1)^{k+1}}{(2 k+1) !}\left(\frac{2 \pi}{5}\right)^{2 k+2} \tau^{-2 k-2} L_{2 k+1}\left(e^{-2 \pi i /(5 \tau)}\right) .
$$

This result shows that there is a "one-to-one correspondence" between identities satisfied by $E_{2 k+2, \chi_{5}}$ and $L_{2 k+1}$ if we know the corresponding behavior of all the functions in the identities under the transformation $\tau \mapsto-1 /(5 \tau)$. We illustrate our observation in the case $k=0$. In this case,

$$
L\left(2, \chi_{5}\right) E_{2, \chi_{5}}\left(e^{2 \pi i \tau}\right)=-\left(\frac{2 \pi}{5}\right)^{2} \tau^{-2} L_{1}\left(e^{-2 \pi i /(5 \tau)}\right) .
$$

From (1-1) and the transformation formula

$$
\eta\left(-\frac{1}{\tau}\right)=\sqrt{-i \tau} \eta(\tau)
$$


we find that

$$
L_{1}\left(e^{-2 \pi i /(5 \tau)}\right)=-\frac{1}{\sqrt{5}} \tau^{2} z\left(e^{2 \pi i \tau}\right),
$$

which upon substitution into (5-4) yields

$$
L\left(2, \chi_{5}\right) E_{2, \chi_{5}}\left(e^{2 \pi i \tau}\right)=\frac{4 \pi^{2}}{25 \sqrt{5}} z\left(e^{2 \pi i \tau}\right) .
$$

Since the $q$-expansions of $E_{2, \chi_{5}}$ and $z(q)$ start with 1, we conclude from (5-5) that

$$
L\left(2, \chi_{5}\right)=\frac{4 \pi^{2}}{25 \sqrt{5}}
$$

and

$$
E_{2, \chi_{5}}(q)=z(q)
$$

The latter is another identity of Ramanujan [Ramanujan 1988, p. 139].

The equivalence of (5-6) and (1-1) was illustrated in [Chan 1996]. However, in that work, the special value $L\left(2, \chi_{5}\right)$ needed to be computed. Here, we obtain the value $L\left(2, \chi_{5}\right)$ as a consequence of the transformation formula of $\eta(\tau)$ and Theorem 5.3.

\section{Acknowledgements}

We sincerely thank Professor Shaun Cooper and the anonymous referee for uncovering several misprints in the earlier version of this paper.

\section{References}

[Bailey 1952a] W. N. Bailey, "A note on two of Ramanujan's formulae", Quart. J. Math., Oxford Ser. (2) 3 (1952), 29-31. MR 13,725d Zbl 0046.04203

[Bailey 1952b] W. N. Bailey, "A further note on two of Ramanujan's formulae", Quart. J. Math., Oxford Ser. (2) 3 (1952), 158-160. MR 14,138f Zbl 0046.27202

[Berndt and Ono 1999] B. C. Berndt and K. Ono, "Ramanujan's unpublished manuscript on the partition and tau functions with proofs and commentary", Sém. Lothar. Combin. 42 (1999), 1-63 (Art. B42c). Reprinted as pp. 39-110 in The Andrews Festschrift, edited by D. Foata and G.N. Han, Springer, Berlin, 2001. MR 2000i:01027 Zbl 0932.11002

[Berndt et al. 2000] B. C. Berndt, H. H. Chan, J. Sohn, and S. H. Son, "Eisenstein series in Ramanujan's lost notebook”, Ramanujan J. 4:1 (2000), 81-114. MR 2001j:11018 Zbl 1024.11026

[Chan 1995] H. H. Chan, "New proofs of Ramanujan's partition identities for moduli 5 and 7", $J$. Number Theory 53:1 (1995), 144-158. MR 96g:11125 Zbl 0844.11064

[Chan 1996] H. H. Chan, "On the equivalence of Ramanujan's partition identities and a connection with the Rogers-Ramanujan continued fraction", J. Math. Anal. Appl. 198:1 (1996), 111-120. MR 96m:11091 Zbl 0858.11056

[Chan and Liu 2003] H. H. Chan and Z.-G. Liu, "Analogues of Jacobi's inversion formula for the incomplete elliptic integral of the first kind", Adv. Math. 174:1 (2003), 69-88. MR 2004b:33027 Zbl 1018.33015 
[Ramanujan 1988] S. Ramanujan, The lost notebook and other unpublished papers, Narosa, New Delhi, and Springer, Berlin, 1988. MR 89j:01078 Zbl 0639.01023

[Serre 1973] J.-P. Serre, A course in arithmetic, Graduate Texts in Math. 7, Springer-Verlag, New York, 1973. MR MR0344216 (49 \#8956) Zbl 0256.12001

[Shen 1994] L.-C. Shen, "On the additive formulae of the theta functions and a collection of Lambert series pertaining to the modular equations of degree 5", Trans. Amer. Math. Soc. 345:1 (1994), 323345. MR 95a:33038 Zbl 0808.33014

[Whittaker and Watson 1927] E. T. Whittaker and G. N. Watson, A course of modern analysis, 4th ed., Cambridge University Press, Cambridge, 1927. MR 97k:01072

Received September 26, 2004. Revised January 16, 2005.

\author{
HENG HuAT CHAN \\ DEPARTMENT OF MATHEMATiCs \\ NATIONAL UNIVERSITY OF SINGAPORE \\ KENT RIDGE, SINGAPORE 119260 \\ REPUBLIC OF SINGAPORE \\ matchh@nus.edu.sg

\section{ZHI-GUO LIU} \\ EAST ChIna Normal University \\ DEPARTMENT OF MATHEMATICS \\ SHANGHAI 200062 \\ P.R. CHINA \\ zgliu@math.ecnu.edu.cn \\ liuzg18@hotmail.com
}

